\title{
Can conventional DWI accurately assess the size of endometrial cancer?
}

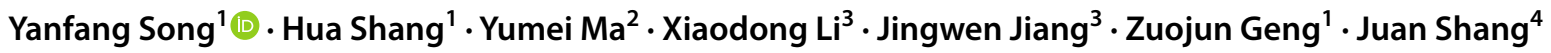

Published online: 12 September 2019

(c) The Author(s) 2019

\begin{abstract}
Purpose To compare T2-weighted image (T2WI) and conventional Diffusion-weighted image (cDWI) of magnetic resonance imaging (MRI) for sensitivity of qualitative diagnosis and accuracy of tumor size (TS) measurement in endometrial cancer (EC). Meanwhile, the effect of the lesion size itself and tumor grade on the ability of T2WI and cDWI of TS assessment was explored. Ultimately, the reason of deviation on size evaluation was studied.

Materials and methods 34 patients with EC were enrolled. They were all treated with radical hysterectomy and performed MR examinations before operation. Firstly, the sensitivity of T2WI alone and T2WI-DWI in qualitative diagnosis of EC were compared according to pathology. Secondly, TS on T2WI and cDWI described with longitudinal (LD) and horizontal diameter (HD) were compared to macroscopic surgical specimen (MSS) quantitatively in the entire lesions and the subgroup lesions which grouped by postoperative tumor size itself and tumor grade. Thirdly, the discrepancy of mean ADC values (ADC mean) and range ADC values (ADC range) between different zones of EC were explored.

Results For qualitative diagnosis, the sensitivity of T2WI-DWI (97\%) was higher than T2WI alone (85\%) $(p=0.046)$. For TS estimation, no significant difference $(\mathrm{PLD}=0.579$; PHD $=0.261)$ was observed between T2WI $(\mathrm{LDT} 2 \mathrm{WI}=3.90 \mathrm{~cm}$; HDT2WI $=2.88 \mathrm{~cm})$ and MSS $(\mathrm{LD}=4.00 \mathrm{~cm} ; \mathrm{HD}=3.06 \mathrm{~cm})$, whereas $\mathrm{TS}$ of $\mathrm{cDWI}(\mathrm{LDDWI}=3.01 \mathrm{~cm} ; \mathrm{HDDWI}=2.54 \mathrm{~cm})$ were smaller than MSS $(\mathrm{PLD}=0.002 ; \mathrm{PHD}=0.002)$ in all lesions. In subgroup of tumor with $\mathrm{G} 1$ (grade 1$)$ and small lesion (defined as maximum diameter $<3 \mathrm{~cm}$ ), both T2WI and cDWI were not significantly different from MSS; In subgroup of tumor with $\mathrm{G} 2+3$ (grade 2 and grade 3 ) and big lesion (maximum diameter $\geq 3 \mathrm{~cm}$ ), T2WI matched well with MSS still, but DWI lost accuracy significantly. The result of ADC values between different zones of tumor showed ADC mean of EC rose from central zone to peripheral zone of tumor gradually and ADC range widened gradually.

Conclusion cDWI can detect EC very sensitively. The TS on cDWI was smaller than the fact for the ECs with G2/3 and big size. The TS of T2WI was in accordance with the actual size for all ECs. The heterogeneity may be responsible for the inaccuracy of cDWI.
\end{abstract}

Keywords Endometrial cancer · Conventional DWI · T2WI · Tumor size

Hua Shang

shanghua519516@sina.com

Yanfang Song

1096450928@qq.com

1 Department of Radiology, The Second Hospital of Hebei Medical University, No. 215, Heping West Road, Xinhua District, Shijiazhuang, Hebei province, China

2 Department of Pathology, The Second Hospital of Hebei Medical University, No. 215, Heping West Road, Xinhua District, Shijiazhuang, Hebei province, China
3 Department of Gynaecology, The Second Hospital of Hebei Medical University, No. 215, Heping West Road, Xinhua District, Shijiazhuang, Hebei province, China

4 Shijiazhuang Institute of Railway Technology, No. 18, Sishuichang Road, Changan District, Shijiazhuang, Hebei province, China 


\section{Introduction}

Endometrial Cancer (EC) is the most common invasive gynecologic malignancy of female genital tract in industrialized countries and slightly secondary to cervical cancer in developing countries [1]. The personalized therapeutic regimen and prognosis for EC are depended on various risk factors: the International Federation of Obstetrics and Gynecology (FIGO) stage, histopathology, grade, and tumor size. A comprehensive and accurate evaluation of the above risks is essential. Magnetic Resonance Imaging (MRI) is a routine preoperative examination for assessing the risks of EC, more important, the diffusion-weighted sequences (DWI) of MRI has been paid more and more attention currently. The excellent capacity of DWI for assessing the above-mentioned risks of EC, such as FIGO stage and histopathology, has been widely studied [2,3]. However, it remains unknown whether DWI can also evaluate the TS precisely or not, which plays an important role among the risks being related with regional and distant metastatic diseases [4-7].

In the present study, we aimed to (a) determine the reliable sequence of MRI reflecting the tumor size of EC through comparing the accuracy of T2WI and cDWI using macroscopic pathological specimens as the gold standard, (b) explore whether the tumor size itself and tumor grade affect the ability of T2WI and cDWI on size measurement or not, and (c) find the reason for reducing the accuracy of cDWI on size estimation: the effect of tumor heterogeneity.

\section{Materials and methods}

This prospective research was approved by the Institutional Review Board and the informed consent from each patient was required.

\section{Patient selection}

Thirty-four consecutive patients with endometrial cancer were enrolled in the present study from March 2016 to November 2017. All clinical, histological, and MRI data from patients were collected and stored in a computerized database. Inclusion criteria were as follows: Radical hysterectomy was performed; MR examination was performed prior to radical hysterectomy within 1 week; carried pathological diagnosis of EC postoperatively; Exclusion criteria: MRI scan was done during more than 1 week before hysterectomy; other cancer-related treatments were performed between MRI scanning and hysterectomy such as radiation, chemotherapy, and hormonotherapy; and postoperative pathology was non-EC, including endometrial hyperplasia accompanied with canceration, endometrial polyp
Table 1 Patients and histopathological tumor characteristics

\begin{tabular}{lll}
\hline Parameters & \\
\hline Age & $\begin{array}{l}\text { Mean: } \\
\text { 59.4 years }\end{array}$ \\
\hline Grading & & \\
G1 & 15 & $38.2 \%$ \\
G2 & 16 & $47.1 \%$ \\
G3 & 3 & $5.9 \%$ \\
Tumor size & & \\
$<3 \mathrm{~cm}$ & 17 & $50 \%$ \\
$\geq 3 \mathrm{~cm}$ & 17 & $50 \%$ \\
\hline
\end{tabular}

accompanied with canceration. The tumor grade and tumor size of all participants were recorded in Pathology Institute and summarized in Table 1.

\section{Imaging acquisition}

Prior to radical hysterectomy, the MRI was performed by Achieva 3.0T Philips MRI machine. All participants were scanned in the prone position with a sixteen-channel SENSE-XL-Torsoa coil. The following parameters were applied: (1) axial spin echoT1-weighted images [repetition time (TR): 400-600 ms, echo time (TE): $10 \mathrm{~ms}$, slice-thickness (ST): $4 \mathrm{~mm}$, intersection gap (SP): $1 \mathrm{~mm}$, number of excitation (NEX): 2 , matrix: $256 \times 128$, field of view (FOV): $24 \mathrm{~cm} \times 24 \mathrm{~cm}$ ) (2) sagittal and oblique axial (perpendicular to the uterine corpus axis) fast spin echo T2-weighted images (TR/TE: 3000-5000/70 ms, ST/SP: 4/1 mm, NEX: 2, matrix: $512 \times 125$, FOV: $24 \mathrm{~cm} \times 24 \mathrm{~cm}$ ) (3) sagittal and oblique axial ssEPI conventional DWI (TR/TE: 6000/88 ms, ST/SP: $4 / 1 \mathrm{~mm}$, two b values: 0 and $1000 \mathrm{~s} / \mathrm{mm}^{2}$, NEX: 2 , matrix: $168 \times 105$, FOV: $31.5 \times 24.2 \mathrm{~cm}$ ). Conventional DWI (cDWI) in the current tial referred image was obtained with full field-of-view (FOV) rather than reduced FOV.

\section{Image analysis}

To avoid bias, the MR images of 34 consecutive patients were assessed by two abdominal experienced radiologists in consensus who were only aware of the clinical symptom without other information (reader 1: 10 years of experience, reader 2: 15 years of experience). Firstly, qualitative diagnoses were determined from T2WI alone and T2WI combined with DWI (T2WI-DWI), respectively. Secondly when EC was confirmed, the TS was measured on T2WI and cDWI respectively. TS were delineated by two dimensions:longitudinal diameter (LD) and horizontal diameter (HD). LD was the largest diameter of lesion from uterus fundus to cervical direction and obtained on sagittal view, HD was the largest diameter from right uterine wall to left uterine wall direction and obtained on oblique axial. The 

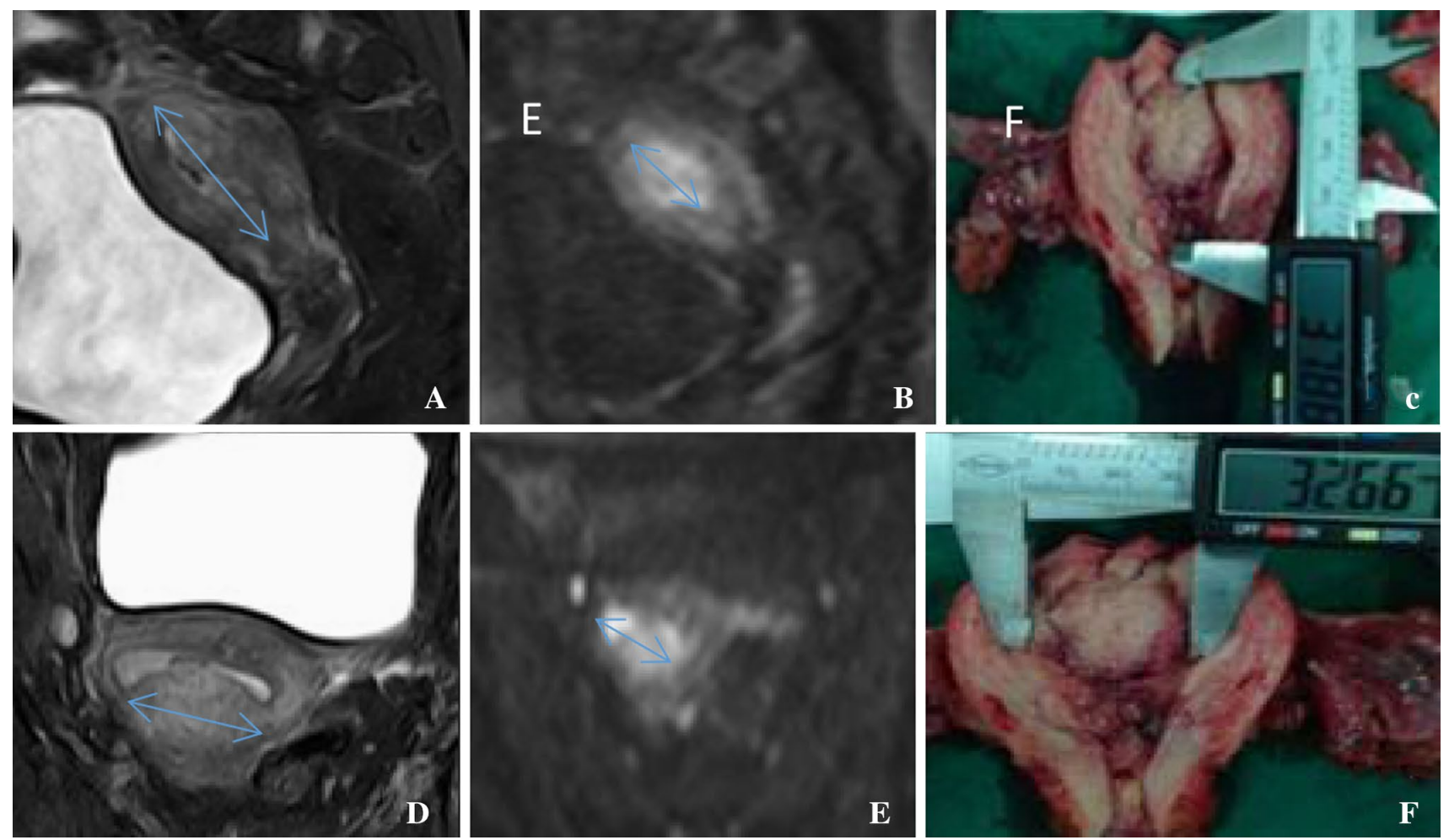

Fig. 1 MR images and macroscopic surgical specimen of a patient with EC (grade 1). a SagT2WI: EC was endometrium thickening with mild hyperintense at posterior wall of uterus, the LDT2WI is $4.31 \mathrm{~cm}$. The evident high signal in front of the uterus is bladder. b Sag DWI: the EC is lesion with hyperintense at posterior wall of uterus, the LDDWI is $2.74 \mathrm{~cm}$. c Specimen: gross at posterior wall with asperous mucosa, the LDPGS is $3.79 \mathrm{~cm}$. d AxiT2WI: gross located on posterior wall with broken mucosa, HDT2WI is $3.43 \mathrm{~cm}$. e AixDWI: lesion with hyperintense at posterior wall of uterus, HDDWI is $3.19 \mathrm{~cm}$. f Specimen: HDPGS is $3.27 \mathrm{~cm}$ maximum LD and HD on preoperative T2WI and DWI were noted as LDT2WI, HDT2WI, LDDWI, HDDWI (Fig. 1).

\section{ADC values measurement}

We measured ADC values of tumors which were larger than $3 \mathrm{~cm}$ as the label of microscopic pathological characteristics of tumor to explore the heterogeneity between different zones of EC. Three different tumor zones were defined in the present study and named peripheral zone,
Fig. 2 oblique aix T2WI and oblique aix ADC of a patient with EC. a The largest contour line of tumor was manually drawn on oblique aix T2WI. Because the TS measured on T2WI was consistent with MSS, the contour line was determined on T2WI firstly. b The contour line was copied onto oblique aix ADC map, then reduce the contour line by $5 \mathrm{~mm}$ and $10 \mathrm{~mm}$, so three closed rings and three zones of tumor were got
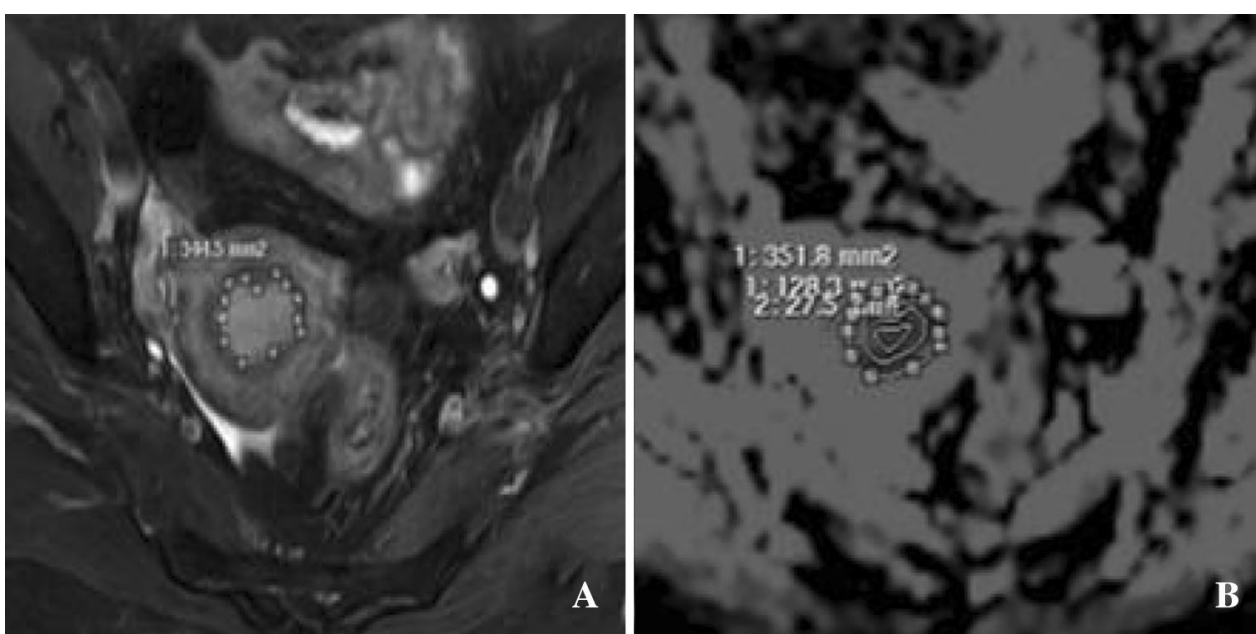
transitional zone, and central zone, respectively. The three different zones were obtained as following: the outmost contour of tumor was manually drawn on oblique axial T2WI primarily, which showed the largest part of tumor. Then the outmost contour was copied to ADC map. Peripheral zone was the band-like area inside $\leq 5 \mathrm{~mm}$ apart from the contour line: 0 to $-5 \mathrm{~mm}$; Transitional zone as the area inside $>5 \mathrm{~mm}$ and $<10 \mathrm{~mm}$ apart from the contour line: $-5 \mathrm{~mm}$ to $-10 \mathrm{~mm}$; Central zone as the area inside $\geq 10 \mathrm{~mm}:-\infty$ to $-10 \mathrm{~mm}$ (Fig. 2). In order to obtain ADC values, two circular regions of interest (ROI) (size: $10 \mathrm{~mm}^{2}$ ) were randomly placed on each zone respectively. Mean ADC values (ADC mean) of each zone were the mean value of two ROIs, range of ADC values (ADC range) were the deviation between maximum and minimum ADC values of two ROIs.

\section{Macroscopic surgical specimen (MSS) assessment}

All patients received radical hysterectomy within 7 days after MR examination; the fresh surgical specimens were collected in the operating room and were anatomized from the anterior uterine wall. On postoperative gross specimens, the LD and HD diameters were obtained with a vernier caliper under assist of pathologists and gynecologists, noted as LD MSS, HDMSS (Fig. 1).

\section{Statistical analysis}

To determine the effect of tumor grade on size assessment of T2WI and cDWI, we divided patients into G1-group $(\leq 5 \%$ solid epithelial component) and G2 +3 -group ( $>5 \%$ solid epithelial component or $<5 \%$ solid growth with marked nuclear atypia); To determine the effect of tumor size itself on size assessment, we divided 34 patients into small-lesion group (maximum diameter of lesion on surgical specimen $<3 \mathrm{~cm}$ ) and big-lesion group (maximum diameter $\geq 3 \mathrm{~cm}$ ). Cases in each group were as Table 1.

Statistical analysis was performed using IBM SPSS Statistics 21.0. The discrepancy of diagnostic sensitivity between T2WI alone and T2WI-DWI was tested using the McNemar test. Scatter graph and Pearson correlation test were used to analyze the correlation of TS between T2WI and MSS, between cDWI and MSS. Wilcoxon test was applied to explore the difference between T2WI and MSS, between cDWI and MSS in all and subgroup lesions respectively. The discrepancy of ADC mean and ADC range among three different zones was examined by Kruskal-Wallis one-way test. A two-tailed $p$ value $<0.05$ was considered statistically significant.

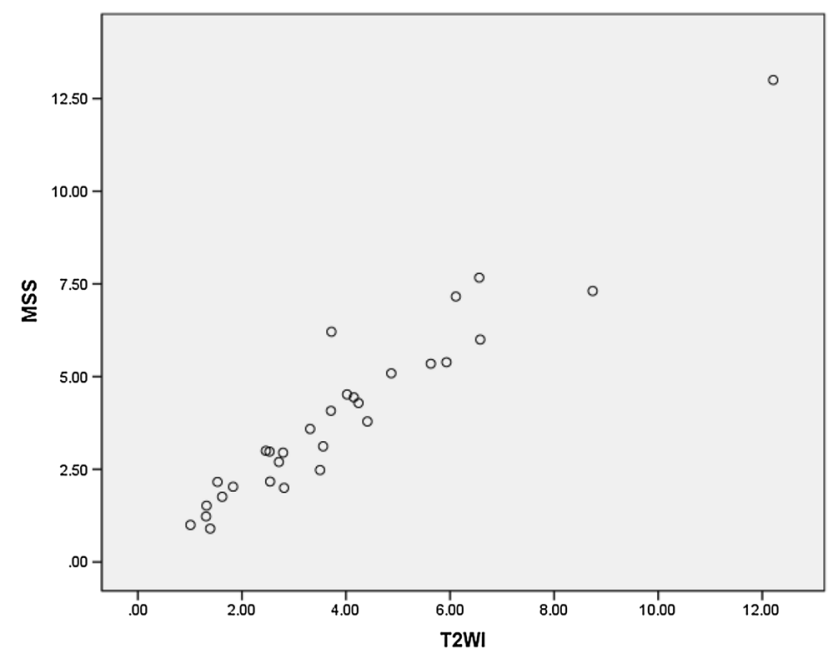

Fig. 3 Scatterplot of longitudinal diameter: T2WI versus MSS ( $\mathrm{x}$ and $y$-axis: size in $\mathrm{cm}$ )

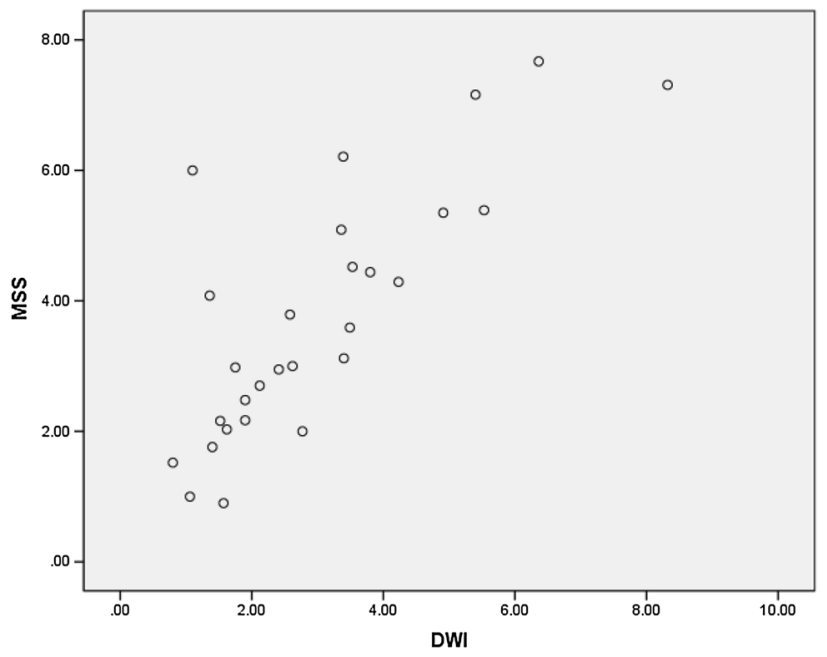

Fig. 4 Scatter plot of longitudinal diameter: DWI versus MSS ( $\mathrm{x}-$ and $\mathrm{y}$-axis: size in $\mathrm{cm}$ )

\section{Results}

\section{Diagnosis sensitivity of MRI: DWI versus T2WI}

In the $34 \mathrm{ECs}$, T2WI alone missed 5 cases, the largest diameters were $0.7 \mathrm{~cm}, 0.9 \mathrm{~cm}, 0.9 \mathrm{~cm}$, and $1.0 \mathrm{~cm}$; T2WI-DWI missed 1 case. The sensitivity of T2WI-DWI (97\%) was higher than that of T2WI alone (85\%), $p=0.046$. 


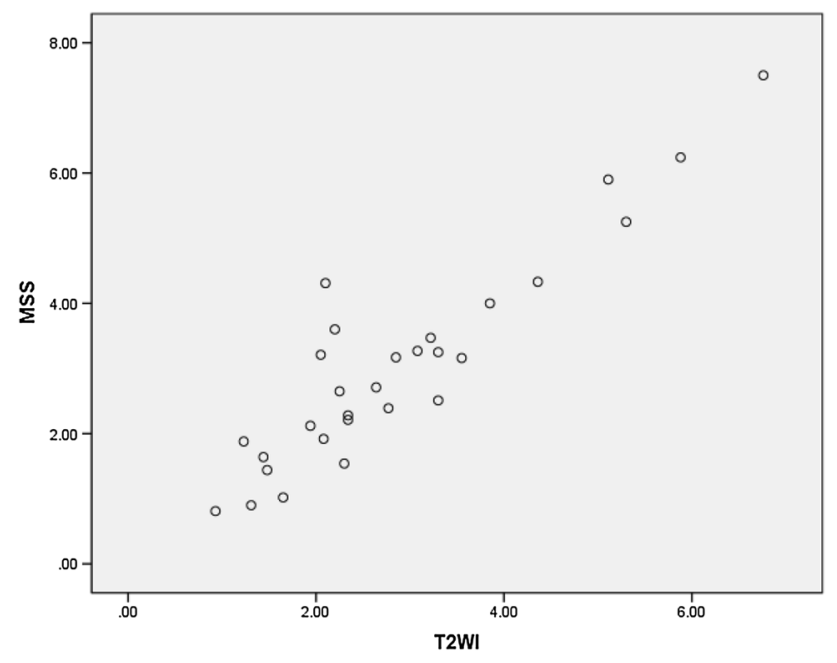

Fig. 5 Scatter plot of horizontal diameter: T2WI versus MSS ( $\mathrm{x}-$ and y-axis: size in $\mathrm{cm}$ )

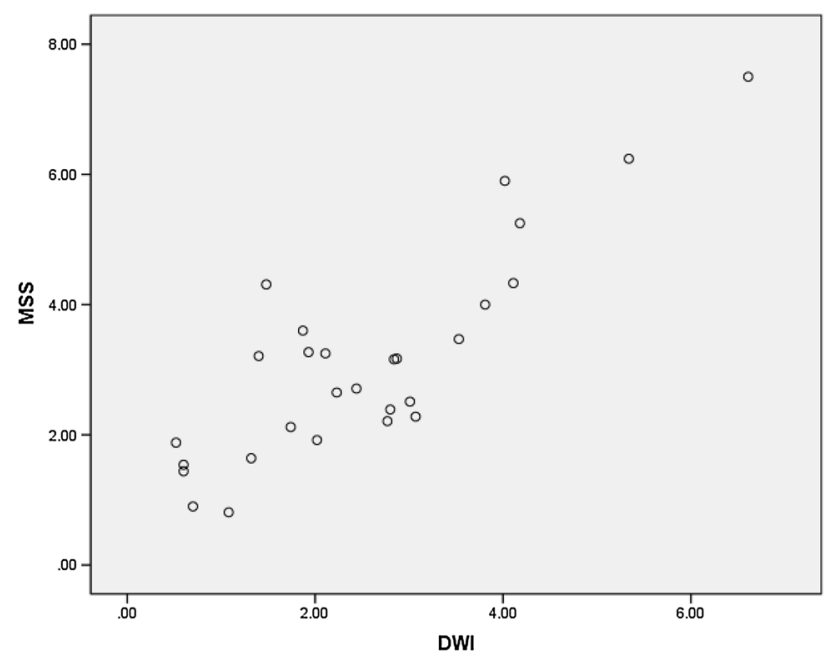

Fig. 6 Scatter plot of horizontal diameter: DWI versus MSS ( $\mathrm{x}-$ and $y$-axis: size in $\mathrm{cm}$ )

Table 2 Wilcoxon test of TS for all the lesions: T2WI versus MSS and $\mathrm{cDWI}$ versus MSS

\begin{tabular}{llllll}
\hline & $\mathrm{LD}(\mathrm{cm})$ & $p$ & $\mathrm{HD}(\mathrm{cm})$ & $p$ \\
\hline T2WI & $3.90 \pm 2.45 \mathrm{~b}(3.53)$ & 0.579 & T2WI & $2.88 \pm 1.44(2.34)$ & 0.261 \\
cDWI & $3.01 \pm 1.80(2.60)$ & 0.002 & cDWI & $2.54 \pm 1.49(2.34)$ & 0.002 \\
MSS & $4.00 \pm 2.56(3.36)$ & & MSS & $3.06 \pm 1.63(2.71)$ & \\
\hline
\end{tabular}

Data are means and standard deviations and data in parenthesis ara medians
Table 3 Wilcoxon test of TS for G1 subgroup: T2WI versus MSS and cDWI versus MSS

\begin{tabular}{llllll}
\hline \multicolumn{2}{c}{ LD $(\mathrm{cm})$} & $p$ & & HD $(\mathrm{cm})$ & $p$ \\
\hline T2WI & $4.16 \pm 3.45(3.15)$ & 0.284 & T2WI & $2.79 \pm 1.73(2.34)$ & 0.162 \\
cDWI & $2.54 \pm 1.74(2.01)$ & 0.575 & cDWI & $2.66 \pm 1.55(2.44)$ & 0.865 \\
MSS & $4.01 \pm 3.85(2.59)$ & & MSS & $2.65 \pm 1.94(2.21)$ & \\
\hline
\end{tabular}

Data are means and standard deviations and data in parenthesis ara medians

Table 4 Wilcoxon test of TS for G2 + 3 subgroup: T2WI versus MSS and $\mathrm{cDWI}$ versus MSS

\begin{tabular}{llllll}
\hline & LD $(\mathrm{cm})$ & $p$ & & HD $(\mathrm{cm})$ & $p$ \\
\hline T2WI & $3.90 \pm 1.34(4.02)$ & 0.151 & T2WI & $2.91 \pm 1.00(2.85)$ & 0.061 \\
cDWI & $3.37 \pm 1.26(3.39)$ & 0.001 & cDWI & $2.53 \pm 1.05(2.44)$ & 0.004 \\
MSS & $4.20 \pm 1.47(4.29)$ & & MSS & $3.32 \pm 1.06(3.21)$ & \\
\hline
\end{tabular}

Data are means and standard deviations and data in parenthesis ara medians

Table 5 Wilcoxon test of TS for small-lesion subgroup: T2WI versus MSS and cDWI versus MSS

\begin{tabular}{llllll}
\hline & LD $(\mathrm{cm})$ & $p$ & & HD $(\mathrm{cm})$ & $p$ \\
\hline T2WI & $2.07 \pm 0.78(1.83)$ & 0.568 & T2WI & $2.01 \pm 0.77(1.94)$ & 0.437 \\
cDWI & $1.74 \pm 0.54(1.69)$ & 0.079 & cDWI & $1.49 \pm 0.85(1.36)$ & 0.059 \\
MSS & $2.00 \pm 0.69(2.03)$ & & MSS & $1.88 \pm 0.79(1.88)$ & \\
\hline
\end{tabular}

Data are means and standard deviations and data in parenthesis ara medians

Table 6 Wilcoxon test of TS for big-lesion subgroup: T2WI versus MSS and cDWI versus MSS

\begin{tabular}{llllll}
\hline & LD $(\mathrm{cm})$ & $p$ & & HD $(\mathrm{cm})$ & $p$ \\
\hline T2WI & $5.31 \pm 2.36(4.44)$ & 0.407 & T2WI & $3.60 \pm 1.38(3.15)$ & 0.134 \\
cDWI & $3.96 \pm 1.83(3.51)$ & 0.009 & cDWI & $3.32 \pm 1.35(2.97)$ & 0.044 \\
MSS & $5.53 \pm 1.48(5.09)$ & & MSS & $3.89 \pm 1.39(3.54)$ & \\
\hline
\end{tabular}

Data are means and standard deviations and data in parenthesis ara medians

\section{Tumor size assessment in all lesions: DWI versus T2WI versus MSS}

Pearson correlation test shew T2WI correlated with a greater coefficient of $\mathrm{r} \mathrm{LD}=0.94$ and $\mathrm{rHD}=0.77(p<0.001)$ respectively, cDWI had a $r=0.74(p<0.001)$ for both LD and HD. Scatter graph was Figs. 3, 4, 5, 6. 
Table 7 Kruskal-Wallis one-way test of ADC mean and ADC range: center versus transition versus periphery

\begin{tabular}{llllll}
\hline & $\begin{array}{l}\text { ADC mean } \\
\left(\times 10^{-6} \mathrm{~mm}^{2} / \mathrm{s}\right)\end{array}$ & $p$ & & $\begin{array}{l}\text { ADC range } \\
\left(\times 10^{-6} \mathrm{~mm}^{2} / \mathrm{s}\right)\end{array}$ & $p$ \\
\hline Center zone & $722.6 \pm 150.2(708.3)$ & 0.280 & Center zone & $88.7 \pm 65.4(84.8)$ & 0.149 \\
Transition zone & $789.2 \pm 174.2(803.8)$ & & Transition zone & $142.5 \pm 88.0(136.6)$ & \\
Center zone & $722.6 \pm 150.2(708.3)$ & 0.004 & Center zone & $88.7 \pm 65.4(84.8)$ & 0.000 \\
Periphery zone & $909.1 \pm 205.0(888.3)$ & & Periphery zone & $319.6 \pm 143.4(386.4)$ & \\
Transition zone & $789.2 \pm 174.2(803.8)$ & 0.055 & Transition zone & $142.5 \pm 88.0(136.6)$ & 0.001 \\
Periphery zone & $909.1 \pm 205.0(888.3)$ & & Periphery zone & $319.6 \pm 143.4(386.4)$ & \\
\hline
\end{tabular}

Data are means and standard deviations and data in parenthesis ara medians

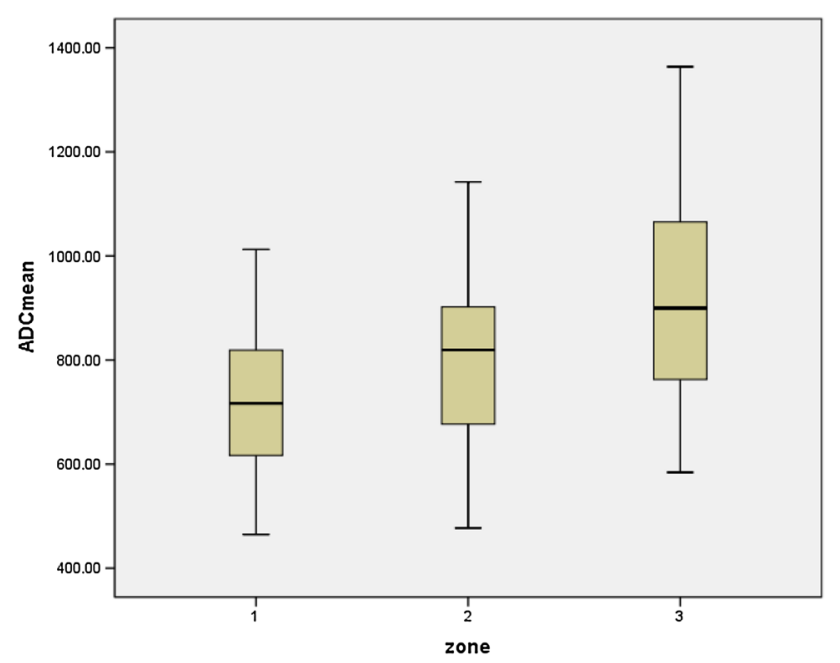

Fig. 7 Comparison of the mean ADC values between central (1), transitional (2) and peripheral (3): the ADCmean increasing from central zone to peripheral zone

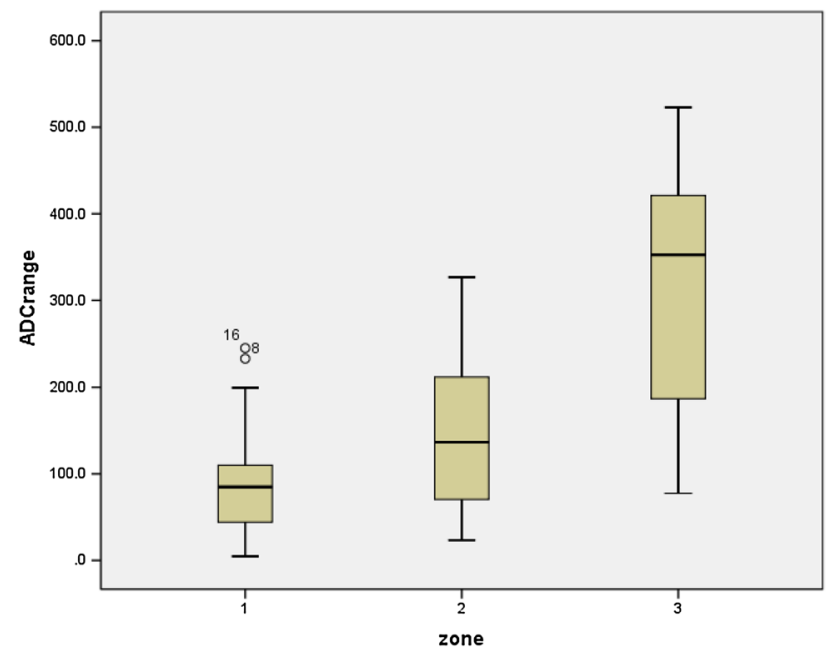

Fig. 8 Comparison of the mean ADC values between central (1), transitional (2) and peripheral (3): the ADC range increasing from central zone to peripheral zone
Wilcoxon test shew no significant difference was observed between T2WI and MSS (PLD $=0.82$ and PHD $=0.88$ respectively). However, diameter of cDWI was significantly smaller than MSS $(\mathrm{PLD}=0.002$ and $\mathrm{PHD}=0.009)$ (Table 2).

\section{Tumor size assessment in subgroups: cDWI versus T2WI versus MSS}

The impact of grade and tumor size itself on accuracy of cDWI and T2WI: for both LD and HD, there was no difference observed between cDWI measurement and MSS, T2WI and MSS in the G1-group and small-lesion group. However, in $\mathrm{G} 2+\mathrm{G} 3$-group and big-lesion group, LD and HD of DWI were smaller than MSS and there was still no difference between T2WI and MSS (Tables 3, 4, 5, 6, 7).

\section{ADC mean and ADC range of EC: central zone versus transitional zone versus peripheral zone}

The ADC mean of central zone, transitional zone, and peripheral zone increased gradually. The ADC range of central zone, transitional zone, peripheral zone widened gradually (Table 5 and Figs. 7, 8).

\section{Discussion}

Many scholars have suggested the TS was an independent predictive of disease recurrence and long-term survival in patients of EC [4-8]. And lots of literatures have also shown that the TS of EC is related to lymph node metastasis and the depth of myometrium involvement [9], which may modify the surgical approach. Thus, an accurate definition of the TS of EC is necessary. Recently, MR examination is the most popular imaging technique to assess the risk of EC, more important T2WI and DWI are the most basic sequences of MR, some researchers have proposed T2WI + DWI can 
reliably replace T2WI + DCE for local staging of EC [10, 11]. Whether MRI can display TS of EC accurately remains unclear. This prospective study thus tried to compare the accuracy of cDWI and T2WI in TS evaluation using surgical specimen as the basis of comparison. We found: (1) cDWI can detect EC lesion very sensitively, but the size of EC measured on cDWI was usually smaller than the fact; (2) The sensitivity of T2WI in qualitative diagnosis of EC is lower than cDWI, but the tumor size of T2WI was in accordance with the actual size well. The largest diameters of lesions which missed by T2WI alone were $0.7 \mathrm{~cm}, 0.9 \mathrm{~cm}$, $0.9 \mathrm{~cm}$ and $1.0 \mathrm{~cm}$ respectively, and yet they were found by T2 WI-DWI. Thus cDWI is more sensitive to small lesions than T2WI. According to prior reports, the sensitivity of cDWI has been considered between $77.7 \%$ and $96 \%$ [12, 13]. The slightly higher sensitivity (97\%) showed in the current study may attribute to fewer cases. Through reviewing literature and measuring ADC value of different areas of the lesions, we summarized two reasons for TS of cDWI smaller than the fact: (1) unclear lesion boundary caused by unsatisfied image quality of cDWI maybe lead to inconsistency between cDWI and the actual; (2) That the heterogeneity of EC itself can result in inhomogeneous signal of cDWI, the tumor boundary was hypointensity relative to tumor interior, makes it difficult to detect the real edge of tumor on cDWI.

To the best of our knowledge, concerning the correlation of MRI different sequences and the pathology in tumor size assessment of EC, there has been only one study published. The study [14] showed reduced field-of-view DWI (rDWI) performed better than T2WI in size evaluation of EC. Different from Priya Bhosale [14], our study focused on the ability of conventional field-of-view DWI (cDWI) and found that tumor size obtained on cFOV DWI yielded a worse correlation with postoperative tumor size than did the T2WI. The cDWI was based on single-shot echo planar imaging (ssEPI) technique which has a longer echo train for phase encoding and is very susceptible to image distortion and artifact from resonance offsets or chemical shift. Nevertheless, rDWI technique in Priya Bhosale's study was based on optimized and constrained undistorted single shot (FOCUS; GE Healthcare, Waukesha) which used a shorter echo train and reduced image distortion and fewer artifacts. Meanwhile, Ota et al. also published cDWI with full FOV yielded more distortion and inferior image quality than rDWI with reduced FOV [15]. The different resolution caused by different FOV and different artifacts may cause different conclusion between the two studies. On the other hand, there are some reports, which illustrated cDWI alone was not as sensitive as cDWI combined with T2WI in judging myometrium involvement of EC [16, 17], also indicated indirectly that cDWI shew the boundary of EC lesion unclear. So an unclear edge of lesion caused by cDWI could be a part of reason for the deviation of TS assessment on cDWI. cDWI covers a larger area than rDWI and can include all lymph node chains in the pelvic cavity, so cDWI is more sensitive to pelvic lymph node metastasis and cDWI is still an indispensable sequence of MRI for EC patients.

Although there is few reports about size assessment of $\mathrm{EC}$, size of other malignancies were broadly discussed. The finding on the accuracy of T2WI is consistent with other carcinomas such as cervical cancer, mammary cancer, nasopharyngeal carcinoma [18-21], in which T2W-MRI has been clearly demonstrated to be a superb imaging procedure in size measurement.

The imaging principle of DWI is based on Brown motion of water molecules; its signal intensity reflects and relies on the microscopic pathological features of tissue. A denser cell population and larger ratio of nucleus to cytoplasm in the tissue can slow the movement of water molecules and this tissue will be hyperintense on DWI. Moreover, the ADC values derived from DWI can quantify these pathological features. As all known, the substantial and disorder cell populations is an essential pathological characteristic of most malignant tumors, so the diffusion rate of water molecules is significantly slow in malignancies and they are always hyperintense on DWI. The heterogeneity is another important pathological feature of cancer, multi-region sequencings performed in many carcinomas including endometrial cancer, breast, lung, colorectal, and renal cancer have uncovered heterogeneity exists in all cancer types [22-27]. The heterogeneity may lead to different diffusion rate of water. Thus, the signal of tumor on DWI is not homogeneous. The present study, which illustrated the mean ADC values of EC increased and the range ADC values widened gradually from the central zone to the peripheral zone of EC and was consistent with previous study on breast cancer [28], actually states that there may be discrepancy of pathological characteristics between interior and periphery of EC. Although this regional heterogeneity of EC has not been confirmed by pathology, David Rew [22] has suggested that most tumors had different peripheral and internal macroscopic pathological features as early as in twentieth century and Stanta G [29] has proposed that the invasion front as compared to the tumor center have different functional properties. Therefore, from these previous reports and the findings in this study, the microscopic pathology of periphery is different from interior in EC can be supposed. This pathologic hypothesis between periphery and interior of EC will be validated in our further research.

The signal intensity of DWI was negatively correlated with ADC value, thus the higher ADC mean and widen ADC range of peripheral tumor would lead hypointense and more heterogenous signal of peripheral part of tumor on DWI. This appearance would cause peripheral tumor is easily neglected when measured on DWI. Therefore, diameter of DWI is often smaller than pathology. This study 
revealed that tumor size of DWI showed no significantly difference from pathology in G1 and small-lesion group but lower accuracy with big tumor group and $\mathrm{G} 2+3$ group. More obvious heterogenicity between periphery and center might exist in bigger and poorly differentiated carcinoma and the peripheral tumor is more easily neglected for these tumors on DWI. Thus, tumor size assessment of poorly differentiated and big lesions in particular needs to use T2WI rather than DWI. These findings are in accord with previous reports concerning breast cancer [30, 31]. Heusinger et al. [30] have suggested that smaller breast cancer were better to assess than larger ones and tumors with a G1 were easier to estimate than tumors with $\mathrm{G} 2 / 3$.

In conclusion, as a kind of functional MR imaging, DWI has a high sensitivity in detecting the discrepancy of micropathology between different tissues, so it has obvious advantage in diagnosing small endometrial carcinoma on early stage. However, the high sensitivity of DWI on the histopathological change of tissue led endometrial cancer, with obvious heterogeneity, is always heterogeneous on DWI. The signal of the inner zone of endometrial carcinoma is often higher than the margin, so when evaluating the tumor size on DWI, the periphery of the lesion might be ignored, resulting in the size of the tumor of DWI smaller than the actual size and this phenomenon was observed in the larger and less differentiated endometrial carcinoma in the present study. Therefore, DWI is superior to T2WI in the qualitative diagnosis of endometrial carcinoma, and T2WI is better than DWI at tumor size measurement.

Open Access This article is distributed under the terms of the Creative Commons Attribution 4.0 International License (http://creativeco mmons.org/licenses/by/4.0/), which permits unrestricted use, distribution, and reproduction in any medium, provided you give appropriate credit to the original author(s) and the source, provide a link to the Creative Commons license, and indicate if changes were made.

\section{References}

1. Terry K L, Missmer S A.Epidemiology of Ovarian and Endometrial Cancers[J].Pathology and Epidemiology of Cancer,2017,6(4):233-246.

2. Kececi I S, Nural M S, Aslan K, et al. Efficacy of diffusionweighted magnetic resonance imaging in the diagnosis and staging of endometrial tumors[J]. Diagnostic \& Interventional Imaging, 2015, 97(2):177-186.

3. Thoeny H C, Froehlich J M, Triantafyllou M, et al. Metastases in normal-sized pelvic lymph nodes: detection with diffusion-weighted MR imaging.[J]. Journal of Urology, 2014, 273(1):125-35.

4. Bourgioti C, Chatoupis K, Tzavara C, et al. Predictive ability of maximal tumor diameter on MRI for high-risk endometrial cancer[J]. Abdominal Radiology, 2016, 41(12):1-12.
5. Yukiharu T,Hidemichi Wi,Kazuhira O,et al. Tumor volume successively reflects the state of disease progression in endometrial cancer[J]. Gynecologic Oncology.2013,129(3):472-477.

6. Julian C. Schink, Alfred W. David Scott M, et al.Tumor Size in Endometrial Cancer[J]. Cancer.1991,67(11):2791-2794.

7. Todo Y, Choi H J, Kang S, et al. Clinical significance of tumor volume in endometrial cancer: A Japan-Korea cooperative study[J]. Gynecologic Oncology, 2013, 131(2):294-298

8. Desimone C, Elder J, Edwards A, et al. Endometrial cancer lesion size is predictive of disease recurrence and long-term survival[J]. Gynecologic Oncology, 2013, 130(1):e71.

9. Ytre-Hauge S, Husby J A, Magnussen I J, et al. Preoperative Tumor Size at MRI Predicts Deep Myometrial Invasion, Lymph Node Metastases, and Patient Outcome in Endometrial Carcinomas[J]. International Journal of Gynecological Cancer, 2015, 25(3):459-466.

10. Bonatti M, Stuefer J, Oberhofer N, et al. MRI for local staging of endometrial carcinoma: Is endovenous contrast medium administration still needed?[J]. European Journal of Radiology, 2015, 84(2):208-214.

11. Mourad A F, Mousa E M. Comparing T2 weighted images/diffusion weighted imaging and $\mathrm{T} 2$ weighted images/dynamic contrast enhanced MRI for endometrial carcinoma myometrial invasion[J]. The Egyptian Journal of Radiology and Nuclear Medicine, 2017, 48(1):323-327.

12. Inada $Y$, Matsuki M, Nakai G, Tatsugami F, Tanikake M,Narabayashi I, et al. Body diffusion-weighted MR imaging of uterine endometrial cancer: is it helpful in the detection of cancer in non-enhanced MR imaging.[J] Eur J Radiol 2009; 70:122-7. Epub 2008 Jan 7.

13. Masroor I, Zeeshan M, Afzal S, et al. Diffusion weighted MR imaging (DWI) and ADC values in endometrial carcinoma[J]. Journal of the College of Physicians and Surgeons-Pakistan : JCPSP, 2010, 20(11):709-713.

14. Bhosale P,Ma J, Iyer R,et al. Feasibility of a reduced field-ofview diffusion-weighted (rFOV) sequence in assessment of myometrial invasion in patients with clinical FIGO stage I endometrial cancer.[J]. Journal of Magnetic Resonance Imaging, 2016, 43(2):316-324.

15. Ota T, Hori M, Onishi H, et al. Preoperative staging of endometrial cancer using reduced field-of-view diffusion-weighted imaging: a preliminary study[J]. European Radiology, 2017.

16. Shen S H, Chiou Y Y, Wang J H, et al. Diffusion-Weighted SingleShot Echo-Planar Imaging with Parallel Technique in Assessment of Endometrial Cancer[J]. American Journal of Roentgenology, 2008, 190(2):481-488.

17. Deng L, Wang Q P, Chen X, et al. The Combination of Diffusion- and T2-Weighted Imaging in Predicting Deep Myometrial Invasion of Endometrial Cancer[J]. Journal of Computer Assisted Tomography, 2015, 39(5):661-673.

18. Hricak H, Lacey C G, Sandles L G, et al. Invasive cervical carcinoma: comparison of MR imaging and surgical findings[J]. Radiology, 1988, 166(3):623-631.

19. Mitchell D G, Snyder B, Coakley F, et al. Early invasive cervical cancer: tumor delineation by magnetic resonance imaging, computed tomography, and clinical examination, verified by pathologic results, in the ACRIN 6651/GOG 183 Intergroup Study.[J]. Journal of Clinical Oncology Official Journal of the American Society of Clinical Oncology, 2006, 24(36):5687-94. 
20. Mann R M, Bult P, van Laarhoven $H$ W, et al. Breast cancer size estimation with MRI in BRCA mutation carriers and other high risk patients.[J]. European Journal of Radiology, 2013, 82(9):1416-22.

21. Popovtzer A, Ibrahim M, Tatro D, et al. MRI to delineate the gross tumor volume of nasopharyngeal cancers: which sequences and planes should be used?[J]. Radiology \& Oncology, 2014, 48(3):323-330.

22. Rew D A. The importance of heterogeneity in tumor pathology[J]. Advances in Anatomic Pathology, 1998, 5(3):156-63.

23. Gatius S, Cuevas D, Fernández C, et al. Tumor Heterogeneity in Endometrial Carcinoma: Practical Consequences[J]. Pathobiology Journal of Immunopathology Molecular \& Cellular Biology, 2017

24. Foulds L. The natural history of cancer. J Chronic Dis. 1958;8:2-37.

25. Kovac M, Navas C, Horswell S, Salm M, Bardella C, Rowan A, et al. Recurrent chromosomal gains and heterogeneous driver mutations characterize papillary renal cancer evolution. Nat Comms. 2015;6:1-11.

26. Yates LR, Gerstung M, Knappskog S, Desmedt C, Gundem G, Van Loo P, etal. Subclonal diversification of primary breast cancer revealed by multiregion sequencing. Nat Med. 2015;21:751-9.

27. Nikbakht H, Panditharatna E, Mikael LG, Li R, Gayden T, Osmond $\mathrm{M}$, et al.Spatial and temporal homogeneity of driver mutations in diffuse intrinsicpontine glioma. Nat Comms. 2016;7:11185.

28. Shin H J, Park J Y, Shin K C, et al. Characterization of tumor and adjacent peritumoral stroma in patients with breast cancer using high-resolution diffusion-weighted imaging: Correlation with pathologic biomarkers[J]. European Journal of Radiology, 2016, 85(5):1004-1011.

29. Stanta G;Jahn SW;Bonin S,etal.Tumour heterogeneity: principles and practical consequences[J].Virchows Arch,2016,469(4):371-184.

30. Heusinger K, Löhberg C, Lux M P, et al. Assessment of breast cancer tumor size depends on method, histopathology and tumor size itself*[J]. Breast Cancer Res Treat, 2005, 94(1):17-23.

31. Mennella S, Garlaschi A, Paparo F, et al. Magnetic resonance imaging of breast cancer: factors affecting the accuracy of preoperative lesion sizing.[J]. Acta Radiologica, 2015, 56(3):260-268.

Publisher's Note Springer Nature remains neutral with regard to jurisdictional claims in published maps and institutional affiliations. 